\title{
PADRÕES E FATORES DETERMINANTES DA ADOÇÃO DE FERRAMENTAS DE GESTÃO DA INOVAÇÃO: UM LEVANTAMENTO COM PRATICANTES NO CONTEXTO DE UM MESTRADO PROFISSIONAL EM INOVAÇÃO
}

\author{
Júlia Araújo Tiso Mudrik (juliatiso@gmail.com) - Universidade Federal de Minas Gerais \\ Paula Gabrielle de Souza Jácome (paulagsj@gmail.com) - Universidade Federal de Minas Gerais \\ Jonathan Simões Freitas (jonathan.ufmg@gmail.com) - Universidade Federal de Minas Gerais \\ Márcio Augusto Gonçalves (marcio@cepead.face.ufmg.br) - Universidade Federal de Minas Gerais \\ Raoni Barros Bagno (rbagno@dep.ufmg.br) - Universidade Federal de Minas Gerais
}

\section{RESUMO}

Em um mercado cada vez mais competitivo e globalizado, a inovação e o desenvolvimento contínuo de novos produtos são atividades essenciais para que as empresas sobrevivam ao longo do tempo. Nesse contexto, várias ferramentas que visam auxiliar e melhorar o desempenho dessas atividades foram desenvolvidas e são utilizadas pelas empresas. É crescente o número de estudos que buscam compreender os padrões (i.e. as ferramentas mais usadas) e os fatores determinantes da adoção dessas ferramentas em diferentes tipos de empresas e países, e a sua relação com o sucesso das inovações. Entre os fatores identificados estão a estratégia de inovação adotada, o grau de formalização do processo, a complexidade da ferramenta, o tamanho da empresa, entre outros. $O$ objetivo deste artigo é ampliar a compreensão sobre os fatores determinantes da adoção das ferramentas de inovação e iniciar a avaliação dos padrões de adoção no contexto brasileiro. Através de um levantamento bibliográfico, as ferramentas e fatores mais citados pela literatura são apresentados. A partir desse levantamento, um questionário foi desenvolvido e aplicado em alunos do programa de mestrado profissional em Inovação Tecnológica e Propriedade Intelectual da UFMG. Constatou-se que as ferramentas mais utilizadas são o Brainstorming, a Gestão de portfólio, o Grupo foco e o Planejamento de cenários. Além disso, algumas das ferramentas mais citadas pela literatura se mostraram desconhecidas para os respondentes.

Palavras chave: Gestão da Inovação; Gestão de Desenvolvimento de Produtos; Ferramentas de Gestão

Área: Ferramentas e métodos de desenvolvimento de produtos e serviços

\section{INTRODUÇÃO}

A gestão da inovação e do desenvolvimentos de produtos é um tópico desafiador em termos teóricos e práticos devido, principalmente, à sua natureza interdisciplinar e aos elevados níveis de incerteza do processo. Diante de um mercado muito competitivo, marcado por constantes mudanças tecnológicas e ciclos de vida de produtos cada vez menores, a atividade de desenvolver novos produtos e serviços tem escalado em sua complexidade e importância (MARKHAM; LEE, 2013). Nesse contexto, para que as empresas se mantenham competitivas e sobrevivam no mercado, a inovação precisa acontecer de forma contínua e sistemática (TIDD et al., 2008). 
As ferramentas e técnicas de gestão são apontadas como uma importante maneira de melhorar e aumentar o sucesso do processo de desenvolvimento produtos (PDP) (NIJSSEN; FRAMBACH, 2000; KRISHNAN; ULRICH, 2001; CHAI; XIN, 2006). Elas são desenvolvidas por consultores, acadêmicos e gestores dentro das próprias organizações (BRADY et al., 1997; ClARK, 1997; PHAAL et al., 2006) e utilizadas para dar suporte às atividades dos diferentes estágios do PDP, incluindo suas fases iniciais.

Diante da importância da inovação e do desenvolvimento de novos produtos e da influência que as ferramentas podem ter nos resultados desses processos, é crescente o número de estudos que buscam compreender os padrões (i.e. as ferramentas mais usadas) e os fatores determinantes da adoção e difusão dessas ferramentas em diferentes tipos de empresas e países. Nesses estudos, o termo adoção se refere a decisão individual de uma empresa em utilizar uma ferramenta ou técnica específica, e o termo difusão refere-se ao número cumulativo de firmas que adotaram as ferramentas ou técnicas ao longo do tempo (NIJSSEN; FRAMBACH, 2000).

O objetivo deste artigo é ampliar a compreensão sobre os fatores de adoção das ferramentas e iniciar a avaliação dos padrões de adoção no contexto brasileiro. Um levantamento bibliográfico das ferramentas e fatores mais citados pela literatura é apresentado no tópico a seguir. A partir desse levantamento, um questionário piloto foi desenvolvido e aplicado em alunos do programa de mestrado profissional em Inovação Tecnológica e Propriedade Intelectual da Universidade Federal de Minas Gerais (UFMG). Os resultados iniciais são apresentados e discutidos no tópico quatro.

\section{FERRAMENTAS DE GESTÃO}

\subsection{Conceito e contexto}

Não existe na academia um conceito bem definido e amplamente utilizado de ferramentas de gestão (BRADY et al., 1997; MORTARA et al., 2014). Brady et al. (1997) destacam que termos como ferramentas, técnicas, métodos, procedimentos, abordagens, entre outros, são normalmente utilizados de forma intercambiável tanto na prática quando na academia. Apesar de alguns autores apresentarem uma diferenciação mais clara entre os termos (e.g. Phaal et al. (2004) que conceituam paradigma, sistema, framework, mapa, modelo, processo, procedimento, técnica e ferramenta), nenhuma delas é amplamente utilizada pela literatura. Tal fato é comprovado pelas próprias definições de ferramentas, que usam termos como técnica e metodologia no texto de seu conceito e, também, pelas listas de ferramentas apresentadas nos estudos, que além de serem diferentes entre si, incluem ferramentas extremamente simples, como brainstorming e checklist, até formas estruturadas, como o Desdobramento da Função Qualidade (em inglês, Quality Function Deployment - QFD), por exemplo.

Entre os trabalhos encontrados sobre ferramentas de gestão, alguns possuem um foco mais genérico (e.g. Rigby (2001)), e outros possuem um domínio específico, como: gestão estratégica (e.g. Clark (1997) e Frost (2003)); gestão de desenvolvimento de produtos (e.g. Nijssen e Frambach (2000) e Tidd e Bodley (2002)); gestão da inovação (e.g. Hidalgo e Albors (2008), Brady et al. (1997) e Phaal et al. (2006)); e previsão (e.g. Popper (2008)). Mais uma vez, a diferenciação do conceito de ferramentas entre esses domínios (i.e. estratégia, inovação, gestão de desenvolvimento de produtos e previsão) não é clara e as listas de ferramentas apresentadas dentro de cada um deles têm muitos itens similares.

Um ponto comum à maior parte dos estudos é o reconhecimento de que uma ampla gama de opções de ferramentas está disponível e é utilizada por gestores com diferentes objetivos e 
para dar suporte a diferentes atividades gerenciais. Elas podem assumir várias formas, incluindo matrizes, tabelas, gráficos, listas de verificação, taxonomias, listas e softwares, juntamente com combinações dessas formas (PHAAL et al., 2006), além de desempenhar um número de diferentes funções, às vezes de forma simultânea. Entre as várias funções e benefícios identificados, estão a geração de informação, o fornecimento de uma estrutura para análise, o incentivo e a facilitação da comunicação de ideias, o auxílio na coordenação e controle, e, também, propósitos simbólicos (CLARK, 1997). Destacam-se, ainda, as possibilidades de compactação e facilitação de visualização de elevado número de informações e a apresentação das informações e pontos complexos de diferentes maneiras, para que novas visões possam ser adquiridas (HUSSEY, 1997; MOISANDER; STENFORS, 2009).

Rigby (2001) coloca que "ferramenta de gestão" pode significar muitas coisas, mas que, na maioria das vezes, envolve um conjunto de conceitos, processos, exercícios e frameworks analíticos. Brady et al. (1997) adotam a definição de que ferramenta de gestão pode ser um documento, abordagem, procedimento, sistema ou método que permite que a empresa alcance ou clarifique um objetivo específico.

Estudos com foco específico em ferramentas de Gestão da Inovação (GI) e Gestão de Desenvolvimento de Produtos (GDP) as definem como uma gama de ferramentas, técnicas e metodologias que ajudam as empresas a se adaptarem às circunstâncias e a enfrentarem os desafios do mercado de forma sistemática, dando suporte às atividades associadas ao processo de inovação (BRADY et al., 1997; PHAAL et al., 2006). Elas vão desde aquelas que visam aumentar a compreensão geral do processo de inovação tecnológica - como modelos conceituais do processo de inovação - até àquelas destinadas a ajudar a gerenciar partes específicas do processo. Elas provêm assistência para diversas decisões estratégicas como: em quais tecnologias investir; a seleção de projetos de pesquisa e desenvolvimento; a seleção de novos produtos; a captação das necessidades dos clientes; a concepção de novos produtos; a promoção da criatividade; e a monitoração e controle de projetos de desenvolvimento (PHAAL et al., 2006). de Waal e Knott (2016) apresentam as ferramentas como qualquer auxílio estruturado, de gestão ou de natureza técnica, utilizado para estruturar ou influenciar a gestão e a execução efetiva do PDP e atividades associadas.

\subsection{Uso das ferramentas de gestão da inovação e do desenvolvimento de produtos e seus fatores determinantes}

Não há na literatura uma lista padrão de ferramentas de GI e GDP. Os diferentes estudos sobre o tema utilizam listas distintas entre si, baseadas nas próprias pesquisa em campo, em revisões detalhadas da literatura e em listas e glossários anteriores. A Tabela 1 apresenta a compilação das ferramentas mais citadas por esses estudos, que não representam, necessariamente, as mais utilizadas. Entraram na compilação todos os artigos encontrados que apresentaram a lista completa de ferramentas utilizada na pesquisa.

Foram encontradas nesse levantamento mais de 180 ferramentas distintas, desconsiderando os casos em que uma mesma ferramenta apareceu com alterações na nomenclaratura, quando identificável. Nijssen e Lieshout (1995) colocam que se todas as versões das ferramentas de desenvolvimento de novos produtos fossem consideradas, mais de 600 tipos poderiam ser identificados. Esse número dá uma ideia da dimensão da quantidade de ferramentas existentes e da diversidade das listas entre os artigos. Apenas uma ferramenta, o QFD, apareceu em todas as listas. Entretanto, essa mesma ferramentas apresenta baixa utilização de acordo com os resultados de vários dos artigos considerados (TIDD; BodLEY, 2002; CHAI; XIN, 2006; LEBER et al., 2014). 
Tabela 1. Ferramentas mais citadas na literatura. Fonte: Elaborada pelos autores.

\begin{tabular}{|c|c|c|c|}
\hline$\#$ & Ferramenta & $\#$ & Ferramenta \\
\hline 1 & QFD & 13 & Gestão de projetos \\
\hline 2 & Brainstorming & 14 & Segmentação \\
\hline 3 & Grupo foco & 15 & Teambuilding \\
\hline 4 & CAD & 16 & Technology watch \\
\hline 5 & Análise conjunta & 17 & Análise de valor \\
\hline 6 & Gestão de portfólio & 18 & Benchmarking \\
\hline 7 & TRIZ & 19 & Concurrent engineering \\
\hline 8 & FMEA & 20 & DFM \\
\hline 9 & DELPHI & 21 & Groupware \\
\hline 10 & Teste de conceito & 22 & Gestão da propriedade intelectual \\
\hline 11 & Prototipagem rápida & 23 & Análise de patentes \\
\hline 12 & Planejamento de cenários & 24 & Workflow \\
\hline
\end{tabular}

Nijssen e Frambach (2000) destacam que a maioria das ferramentas foram criadas para lidar com diferentes tipos de problemas e, portanto, para uso em diferentes estágios do processo de desenvolvimento. Entretanto, na prática, o uso não é tão focado, e observa-se que algumas ferramentas possuem mais de uma função e são utilizadas em mais de um estágio (NIJSSEN; LIESHOUT, 1995; CHAI; XIN, 2006).

Alguns estudos apresentam uma categorização das ferramentas. González e Palacios (2002), por exemplo, as dividem em cinco categorias genéricas: técnicas de design, técnicas organizacionais, técnicas de fabricação, tecnologias da informação e envolvimento do fornecedor. Hidalgo e Albors (2008) as classificam em dez tipos de acordo com os problemas de negócio das organizações, destacando que a correlação entre o problema empresarial específico de uma empresa e a metodologia que o resolve não é totalmente clara. Tidd e Thuriaux (2016) agruparam as ferramentas em oito funções de acordo com o processo de inovação: Estratégia de inovação; Inteligência de negócios externa; Gerenciamento de ideias; Gerenciamento de portfólio de produtos; Gestão de portfólio de tecnologia; Desenvolvimento e lançamento; Pós-lançamento; Gestão de recursos e competências.

Além de levantarem quais ferramentas são as mais utilizadas, alguns trabalhos também investigam quais fatores influenciam diretamente a adoção e difusão das mesmas. Nijssen e Frambach (2000), por exemplo, realizaram um survey com 70 indústrias holandesas e, através da análise de regressão, avaliaram a influência do tamanho da empresa, do nível de envolvimento da alta administração, das características do processo de PDP (i.e. número de estágios, número de departamentos envolvidos, nível de comunicação entre departamentos), do uso anterior de ferramentas e dos objetivos relacionados à inovação com a adoção de ferramentas. Os resultados apontaram que os fatores significativos foram o nível de comunicação entre departamentos, o número de estágios do processo, os objetivos relacionados à inovação e o uso anterior de ferramentas. O tamanho da empresa não apresentou significância e o nível de envolvimento da alta administração, uma influência muito baixa. Para esse fator, os autores destacaram que o resultado vai contra os trabalhos anteriores e indicam que sua forma de medição poderia ter sido melhor e mais direta.

Os resultados da pesquisa realizada por Tidd e Bodley (2002) em 50 projetos de desenvolvimento de produtos em 25 empresas corroboram com Nijssen e Frambach (2000). Os autores identificaram que o grau de inovação dos projetos influenciam tanto a frequência 
de uso quanto a percepção de sucesso no uso das ferramentas. Chai e Xin (2006), a partir de uma pesquisa realizada em empresas de Cingapura, também encontraram os objetivos relacionados à inovação como um fator influente do uso, mas colocam que mais críticos do que ele são o tamanho da empresa e principalmente o suporte da alta gestão.

A tabela 2, com estrutura baseada em Hutzschenreuter e Kleindienst (2006), apresenta uma compilação desses trabalhos para sintetizar e contextualizar os fatores identificados como influenciadores do uso de ferramentas de GI e GDP pelas organizações. Além dos trabalhos empíricos, foram considerados também trabalhos teóricos que contribuem diretamente com o tema.

Tabela 2. Fatores explicativos do uso de ferramentas de gestão da inovação e desenvolvimento de produtos: compilação da literatura. Fonte: Elaborada pelos autores.

\begin{tabular}{|c|c|c|}
\hline \multicolumn{2}{|c|}{ FATOR } & REFERÊNCIAS \\
\hline \multirow{5}{*}{$\begin{array}{l}\text { Características da } \\
\text { organização }\end{array}$} & Tamanho & $\begin{array}{l}\text { (NIJSSEN; FRAMBACH, 2000); (DE WAAL; KNOTT, } \\
\text { 2016);(CHAI, 2006) }\end{array}$ \\
\hline & Idade & (DE WAAL; KNOTT, 2016) \\
\hline & $\begin{array}{l}\text { Experiência/Uso anterior } \\
\text { de ferramentas }\end{array}$ & $\begin{array}{l}\text { (NIJSSEN; FRAMBACH, 2000); (MARKHAM; LEE, } \\
2013 \text { ) }\end{array}$ \\
\hline & $\begin{array}{l}\text { Pessoal qualificado / } \\
\text { Conhecimento }\end{array}$ & $\begin{array}{l}\text { (HIDALGO, 2008); (MARKHAM; LEE, 2013); (LEBER } \\
\text { et al., 2014) }\end{array}$ \\
\hline & Cultura & $\begin{array}{l}\text { (HIDALGO, 2008); (LIBUTTI, 2000); (GONZÁLEZ, } \\
\text { 2000) }\end{array}$ \\
\hline Contexto estratégico & $\begin{array}{l}\text { Objetivos estratégicos / } \\
\text { Nível das inovações }\end{array}$ & $\begin{array}{l}\text { (DE WAAL; KNOTT, 2016); (TIDD; BODLEY, 2002); } \\
\text { (CHAI, 2006); (NIJSSEN; FRAMBACH, 2000) }\end{array}$ \\
\hline Contexto ambiental & Setor & $\begin{array}{l}\text { (DE WAAL; KNOTT, 2016); (TIDD; THURIAUX, } \\
\text { 2016) }\end{array}$ \\
\hline $\begin{array}{l}\text { Características do } \\
\text { problema }\end{array}$ & Tipo de tarefa/problema & (HIDALGO, 2008) \\
\hline \multirow{4}{*}{$\begin{array}{l}\text { Características do } \\
\text { processo }\end{array}$} & Nível de formalização & $\begin{array}{l}\text { (DE WAAL; KNOTT, 2016); (MARKHAM; LEE, } \\
\text { 2013); (NIJSSEN; FRAMBACH, 2000) }\end{array}$ \\
\hline & Apoio top-down & $($ NIJSSEN; FRAMBACH, 2000)*; (CHAI, 2006) \\
\hline & Tamanho do time & (DE WAAL; KNOTT, 2016) \\
\hline & $\begin{array}{l}\text { Complexidade do } \\
\text { processo }\end{array}$ & (D'ALVANO; HIDALGO, 2012) \\
\hline \multirow{3}{*}{$\begin{array}{l}\text { Características das } \\
\text { ferramentas }\end{array}$} & Utilidade & (CHAI, 2006); (LEBER et al., 2014) \\
\hline & Popularidade & (D'ALVANO; HIDALGO, 2012); (LEBER et al., 2014) \\
\hline & $\begin{array}{l}\text { Complexidade/Facilidade } \\
\text { de uso }\end{array}$ & $\begin{array}{l}\text { (D'ALVANO; HIDALGO, 2012); (LEBER et al., } \\
\text { 2014);(CHAI, 2006) }\end{array}$ \\
\hline
\end{tabular}

Observa-se que os fatores levantados estão relacionados, principalmente, às características da organização, aos objetivos estratégicos, ao nível de formalização do processo e à facilidade de uso da ferramenta.

\section{METODOLOGIA}

Com o objetivo de iniciar a avaliação dos padrões e fatores determinantes da adoção de ferramentas de gestão da inovação e desenvolvimento de produtos no contexto brasileiro, foi desenvolvido, a partir da revisão da literatura, um questionário-piloto para ser aplicado em alunos do programa de mestrado profissional em Inovação Tecnológica e Propriedade 
Intelectual da UFMG. O programa foi escolhido por concentrar um número representativo de profissionais envolvidos com o ambiente de gestão de inovação das empresas.

A revisão da literatura teve como principais objetivos: (1) definir a lista de ferramentas que seria avaliada; e (2) identificar os fatores indicados pela literatura como determinantes da adoção das ferramentas. Para limitar o tamanho do questionário, a pesquisa foi restringida a onze ferramentas que foram descritas em um glossário, seguindo orientação de Nijssen e Frambach (2000). Cada fator identificado se transformou em pelo menos uma pergunta do questionário, formatadas de acordo com a literatura e pesquisas anteriores. Na revisão, foram avaliados artigos da base de dados do Web of Knowledge que possuem em seu tópicos os termos New Product Development Technique*, New Product Development Tool*, Innovation Management Technique*, ou Innovation Management tool*.

O procedimento de coleta de dados se deu através de questionários padronizados disponibilizados em um link no Google Docs enviados por email para os alunos do programa de mestrado profissional, incluindo aqueles de disciplinas isoladas. Foram obtidas 33 respostas válidas, entre as quais 18 respondentes eram regularmente matriculados e o restante cursavam pelo menos uma disciplina isolada. Todos eles trabalhavam ou já haviam trabalhado em pelo menos uma organização e $76 \%$ exerciam atividades com relação à gestão da inovação da empresa. Entre os respondentes, 4 (12\%) possuíam cargo de gerência sênior, 9 (27\%) de gerência média e 17 (52\%) profissional. Em relação à área de formação, 70\% possuíam pelo menos uma formação em ciências gerenciais (i.e. economia, administração, ciências contábeis, engenharia de produção, ciência da informação). A tabela 3 apresenta as informações sobre o setor e tamanho das empresas associadas a amostra. Entre elas, 48\% afirmam não ter uma abordagem padrão para a GI; $27 \%$ afirmam que embora não seja seguido nenhum processo formalmente documentado, a empresa segue uma trajetória clara de tarefas a serem cumpridas no desenvolvimento de produtos; e $24 \%$ afirma ter um processo formalmente documentado.

Tabela 3. Característica da amostra - Empresas em que os respondentes trabalham ou trabalhavam. Fonte: Elaborada pelos autores.

\begin{tabular}{l|c|c|c|c|c|c} 
& \multicolumn{2}{|c|}{ Total } & $\begin{array}{c}\text { Até 19 } \\
\text { empregados }\end{array}$ & $\begin{array}{c}\text { 20 a 99 } \\
\text { empregados }\end{array}$ & $\begin{array}{c}\text { 100 a 499 } \\
\text { empregados }\end{array}$ & $\begin{array}{c}\text { Mais de 500 } \\
\text { empregados }\end{array}$ \\
\hline Serviço & 21 & $64 \%$ & 8 & 3 & 3 & 7 \\
\hline Indústria & 6 & $18 \%$ & 1 & - & - & 5 \\
\hline Outros & 6 & $18 \%$ & 1 & 1 & 2 & 2
\end{tabular}

\section{RESULTADOS E DISCUSSÃO}

A tabela 4 a seguir apresenta os percentuais de uso e conscientização dos respondentes em relação às onze ferramentas listadas no questionário. A frequência de uso e a média da utilidade percebida para cada uma delas também são exibidas.

Da lista de onze ferramentas, quatro delas são ou já foram utilizadas por mais de $50 \%$ dos respondentes (Brainstorming, Gestão de portfólio, Grupo foco e Planejamento de cenários). Elas também representam as ferramentas que os respondentes possuem maior conscientização (menor taxa de resposta "Nunca escutei falar"). Observa-se que a TRIZ, seguida do CAD e FMEA, representam as ferramentas menos conhecidas pela amostra (taxa de resposta "Nunca escutei falar" de $36 \%$ e $27 \%$, respectivamente) e também estão na lista das menos usadas. 
Tabela 4. Adoção e utilidade percebida das ferramentas de Gestão da Inovação de Desenvolvimento de Produtos. Fonte: Elaborada pelos autores.

\begin{tabular}{l|c|c|c||c|c||c} 
& $\begin{array}{c}\text { Uso ou Já } \\
\text { usei }\end{array}$ & $\begin{array}{c}\text { Já escutei } \\
\text { falar, mas } \\
\text { nunca usei }\end{array}$ & $\begin{array}{c}\text { Nunca } \\
\text { escutei falar }\end{array}$ & $\begin{array}{c}\text { Uso com } \\
\text { frequência }\end{array}$ & $\begin{array}{c}\text { Uso } \\
\text { raramente }\end{array}$ & $\begin{array}{c}\text { Utilidade } \\
\text { (0 a 5) }\end{array}$ \\
\hline Brainstorming & $\mathbf{8 8 \%}$ & $12 \%$ & $0 \%$ & $50 \%$ & $50 \%$ & 3,7 \\
\hline Gestão de portfólio & $\mathbf{6 4 \%}$ & $33 \%$ & $3 \%$ & $\mathbf{7 9 \%}$ & $21 \%$ & 4,2 \\
\hline Grupo foco & $\mathbf{6 1 \%}$ & $36 \%$ & $3 \%$ & $21 \%$ & $\mathbf{7 9 \%}$ & 3,7 \\
\hline $\begin{array}{l}\text { Planejamento de } \\
\text { Cenários }\end{array}$ & $\mathbf{5 5 \%}$ & $33 \%$ & $12 \%$ & $\mathbf{6 7 \%}$ & $33 \%$ & 3,8 \\
\hline Teste de conceito & $\mathbf{4 5 \%}$ & $42 \%$ & $12 \%$ & $44 \%$ & $\mathbf{5 6 \%}$ & 3,9 \\
\hline Análise conjunta & $33 \%$ & $\mathbf{4 5 \%}$ & $21 \%$ & $\mathbf{6 9 \%}$ & $31 \%$ & 4,3 \\
\hline QFD & $27 \%$ & $\mathbf{6 7 \%}$ & $6 \%$ & $0 \%$ & $\mathbf{1 0 0 \%}$ & 3,1 \\
\hline FMEA & $18 \%$ & $\mathbf{5 5 \%}$ & $27 \%$ & $33 \%$ & $\mathbf{6 7 \%}$ & 3,5 \\
\hline CAD & $15 \%$ & $\mathbf{5 8 \%}$ & $27 \%$ & $0 \%$ & $\mathbf{1 0 0 \%}$ & 3,0 \\
\hline TRIZ & $15 \%$ & $\mathbf{4 8 \%}$ & $36 \%$ & $\mathbf{7 1 \%}$ & $\mathbf{2 9} \%$ & 4,1 \\
\hline DELPHI & $9 \%$ & $\mathbf{7 3 \%}$ & $18 \%$ & $40 \%$ & $\mathbf{6 0 \%}$ & 4,2
\end{tabular}

Como a lista de ferramentas utilizadas nos estudos anteriores são distintas entre si, a comparação direta entre as mais usadas não é possível. Entretanto, destaca-se que o Brainstorming é a ferramenta mais usada sempre em que está presente na lista (e.g. Nijssen e Lieshout (1995), Chai e Xin (2006) e D'Alvano e Hidalgo (2012)). Outras ferramentas que aparecem entre as mais usadas nos trabalhos anteriores são: Teste de Conceito e Teste inhome (NIJSSEN; LIESHOUT, 1995); Benchmarking, DOE e FMEA (CHAI; XIN, 2006); CAD e CAE (GONZÁLEZ, 2000); Estudo de tendências, Alianças estratégicas, Documentação de processo, Análise de valor, Benchmarking, Gráfico Gantt e Plano de Negócios (D'Alvano; Hidalgo, 2012). Observa-se que, contrariamente ao encontrado nesta pesquisa, o CAD e FMEA aparecem como as ferramentas mais utilizadas em dois dos artigos pesquisados. Uma possível justificativa é que a amostra desse estudo tem em sua maioria empresas de serviço, enquanto os outros estudos focam em indústrias de manufatura.

O número médio de ferramentas utilizadas pela amostra foi de 3,25 , um pouco maior do que o encontrado por Nijssen e Frambach (2000) (média de 3) e menor do que o encontrado por Chai e Xin (2006) (média de 4). Os respondentes com cargo de gerência sênior apresentaram uma média de 5,25 ferramentas, os de gerência média 3,22 e os de nível profissional 2,88. Esse dado indica que pode haver um diferença na adoção de acordo com o nível gerencial do cargo.

Após a avaliação da lista de ferramentas descritas no questionário, os respondentes puderam listar outras ferramentas que faziam ou já fizeram uso. Foram citadas 27 ferramentas. Entre elas, o Technology Roadmapping (TRM) foi o mais citado (5 respondentes), seguido pela análise SWOT (4 respondentes), Design Thinking (3), Canvas (3) e Scrum (2) e Plano de Negócios (2). Nota-se que nenhuma delas está na lista das mais citadas pela literatura.

A tabela 5 apresenta em qual etapa do processo de inovação os respondentes que usam ou já utilizaram as ferramentas as empregaram. Os resultados mostram um uso desfocado da maioria das ferramentas. Nota-se que três das quatro ferramentas com mais de $50 \%$ de uso são mais utilizadas na fase de Avaliação e Seleção de ideias. Além disso, mais da metade das ferramentas pesquisadas são mais usadas na fase de Desenvolvimento. Destaca-se também que nenhuma das onze ferramentas pesquisadas é mais empregada na fase de Difusão/Marketing/Vendas. 
Tabela 5. Adoção das ferramentas por etapa do processo de Gestão da Inovação. Fonte: Elaborada pelos autores.

\begin{tabular}{l|c|c|c|c} 
& $\begin{array}{c}\text { Geração de } \\
\text { ideias }\end{array}$ & $\begin{array}{c}\text { Avaliação/Seleção } \\
\text { de ideias }\end{array}$ & Desenvolvimento & $\begin{array}{c}\text { Difusão/Marketing } \\
\text { Nendas }\end{array}$ \\
\hline CAD & $0 \%$ & $0 \%$ & $\mathbf{1 0 0 \%}$ & $0 \%$ \\
\hline DELPHI & $0 \%$ & $0 \%$ & $\mathbf{1 0 0 \%}$ & $0 \%$ \\
\hline Teste de conceito & $7 \%$ & $13 \%$ & $\mathbf{7 3} \%$ & $7 \%$ \\
\hline FMEA & $17 \%$ & $17 \%$ & $\mathbf{6 7 \%}$ & $0 \%$ \\
\hline QFD & $11 \%$ & $11 \%$ & $\mathbf{5 6 \%}$ & $22 \%$ \\
\hline TRIZ & $25 \%$ & $25 \%$ & $\mathbf{5 0 \%}$ & $0 \%$ \\
\hline Gestão de portfólio & $0 \%$ & $\mathbf{4 8 \%}$ & $30 \%$ & $22 \%$ \\
\hline Planejamento de & $6 \%$ & $\mathbf{3 9} \%$ & $22 \%$ & $33 \%$ \\
Cenários & $20 \%$ & $\mathbf{5 5 \%}$ & $20 \%$ & $5 \%$ \\
\hline Grupo foco & $13 \%$ & $\mathbf{6 3} \%$ & $13 \%$ & $13 \%$ \\
\hline Análise conjunta & $\mathbf{8 6 \%}$ & $3 \%$ & $7 \%$ & $3 \%$
\end{tabular}

\section{CONCLUSÃO}

O objetivo deste artigo foi ampliar a compreensão sobre os fatores de adoção das ferramentas de gestão da inovação e desenvolvimento de produtos e iniciar a avaliação dos padrões de adoção no contexto brasileiro. Nessa primeira rodada do questionário piloto, foi possível testar o instrumento e fazer algumas constatações importantes sobre a amostra e também sobre a literatura.

Através da revisão bibliográfica dos artigos sobre o tema, observou-se que não há uma lista bem definida de ferramentas de gestão da inovação e desenvolvimento de produtos e que falta uma nomenclatura padrão para algumas delas. Além disso, os diferentes estudos sobre o tema apresentam diversos fatores que influenciam a adoção, incluindo características da empresa, do processo e das próprias ferramentas.

Em relação a adoção das ferramentas pela amostra, a partir de análise descritivas dos dados, constatou-se que o Brainstorming, a Gestão de portfólio, o Grupo foco e o Planejamento de cenários são as mais utilizadas. Algumas das ferramentas mais citadas pela literatura apresentaram-se consideravelmente desconhecidas pelos respondentes, como a TRIZ, CAD, FMEA e Análise conjunta. Algumas das ferramentas, apesar dos respondentes serem conscientes da sua existência, apresentam taxa de utilização muito baixas, como o QFD e o DELPHI. Além disso, ferramentas não presentes na lista foram citadas por um número significativo de respondentes, como o TRM, a análise SWOT e o Design thinking, e devem ser consideradas em pesquisas futuras.

Utilizando-se desses resultados iniciais, estudo futuros podem ser realizados com amostras mais amplas para que outras análises estatísticas possam ser realizadas no contexto brasileiro (e.g. análise de regressão) para identificar quais fatores influenciam na adoção e quais práticas estão sendo adotadas com sucesso nas diferentes etapas do processo de inovação. Trabalhos com este foco contribuem diretamente com as dificuldades encontradas pelas empresas em identificar, selecionar, adotar e integrar as ferramentas dentro de seus processos e sistemas organizacionais (PHAAL et al., 2006). 


\section{REFERÊNCIAS}

BRADY, T. et al. Tools for technology management: an academic perspective. Technovation, v. 17, n. 8, p. 417-426, 1997. ISSN 0166-4972.

CHAI, K.H.; XIN, Y. The application of new product development tools in industry: the case of Singapore. IEEE Transactions on Engineering Management, v. 53, n. 4, p. 543-554, 2006. ISSN 0018-9391.

CLARK, D.N. Strategic management tool usage: a comparative study. Strategic Change, v. 6, n. 7, p. 417-427, 1997. ISSN 1099-1697.

D'ALVANO, L.; HIDALGO, A. Innovation management techniques and development degree of innovation process in service organizations. R\&D Management, v. 42, n. 1, p. 60-70, 2012. ISSN 1467-9310.

DE WAAL, G.A.; KNOTT, P. Patterns and drivers of NPD tool adoption in small hightechnology firms. IEEE Transactions on Engineering Management, v. 63, n. 4, p. 350-361, 2016. ISSN 0018-9391.

FROST, F.A. The use of strategic tools by small and medium-sized enterprises: an Australasian study. Strategic change, v. 12, n. 1, p. 49-62, 2003. ISSN 1099-1697.

GONZÁLEZ, F.J.M.; PALACIOS, T.M.B. The effect of new product development techniques on new product success in Spanish firms. Industrial Marketing Management, v. 31, n. 3, p. 261-271, 2002. ISSN 0019-8501.

HIDALGO, A.; ALBORS, J. Innovation management techniques and tools: a review from theory and practice. R\&d Management, v. 38, n. 2, p. 113-127, 2008. ISSN 1467-9310.

HUSSEY, D.E. Glossary of techniques for strategic analysis. Strategic Change, v. 6, n. 2, p. 97-115, 1997. ISSN 1099-1697.

HUTZSCHENREUTER, T.; KLEINDIENST, I. Strategy-process research: what have we learned and what is still to be explored. Journal of Management, v. 32, n. 5, p. 673-720, 2006. ISSN 0149-2063.

KRISHNAN, V.; ULRICH, K.T. Product development decisions: A review of the literature. Management science, v. 47, n. 1, p. 1-21, 2001. ISSN 0025-1909.

LEBER, M.; BASTIČ, M.; BUCHMEISTER, B. The trends in usage and barriers of innovation management techniques in new product development. Strojniški vestnik-Journal of Mechanical Engineering, v. 60, n. 6, p. 382-388, 2014. ISSN 0039-2480.

MARKHAM, S.K.; LEE, H. Product development and management association's 2012 comparative performance assessment study. Journal of Product Innovation Management, v. 30, n. 3, p. 408-429, 2013. ISSN 1540-5885.

MOISANDER, J.; STENFORS, S. Exploring the edges of theory-practice gap: Epistemic cultures in strategy-tool development and use. Organization, v. 16, n. 2, p. 227-247, 2009. ISSN 1350-5084.

MORTARA, L. et al. Tool fingerprinting: Characterising management tools. International Conference on Management of Engineering \& Technology (PICMET), 2014, 2014. Portland IEEE. p.102-117. 
NIJSSEN, E.J.; FRAMBACH, R.T. Determinants of the adoption of new product development tools by industrial firms. Industrial Marketing Management, v. 29, n. 2, p. 121131, 2000. ISSN 0019-8501.

NIJSSEN, E.J.; LIESHOUT, K.F.M. Awareness, use and effectiveness of models and methods for new product development. European Journal of Marketing, v. 29, n. 10, p. 27-44, 1995. ISSN 0309-0566.

PHAAL, R.; FARRUKH, C.J.P.; PROBERT, D.R. A framework for supporting the management of technological knowledge. International Journal of Technology Management, v. 27, n. 1, p. 1-15, 2004. ISSN 0267-5730.

Technology management tools: concept, development and application. Technovation, v. 26, n. 3, p. 336-344, 2006. ISSN 0166-4972.

POPPER, R. How are foresight methods selected? Foresight, v. 10, n. 6, p. 62-89, 2008. ISSN 1463-6689.

RIGBY, D.K. Putting tools to the test: senior executives rate 25 top management tools. Strategy \& Leadership, v. 29, n. 3, p. 4-12, 2001. ISSN 1087-8572.

TIDD, J.; BESSANT, J.; PAVITT, K. Gestão da Inovação. Porto Alegre: Bookman, 2008. 600p. ISBN 9788577802029.

TIDD, J.; BODLEY, K. The influence of project novelty on the new product development process. 2002.

TIDD, J.; THURIAUX, A.B. Innovation management practices: cross-sectorial adoption, variation, and effectiveness. R\&D Management, 2016. ISSN 1467-9310. 